\title{
Exploring User-Centered Intelligent Road Lighting Design: A Road Map and Future Research Directions
}

\author{
Çağdaş Atıcı, Member, IEEE, Tanır Özçelebi, Member, IEEE and Johan J. Lukkien, Member, IEEE
}

\begin{abstract}
Traditional road lighting systems are outdated and should be replaced with systems which can sense their environment, i.e., users and their consumer electronics (CE) devices, and react intelligently. Realizing such a system requires an interdisciplinary approach which analyzes the efficient system architecture and communication technology while taking into account the user needs. To understand these necessities, subjective experiments should be conducted in a practical testbed, similar to ours. In this paper, we first present the state-of-the-art solutions in the literature. Then, we describe the system architecture of our testbed deployed on a real street along with the preliminary experiments. Finally, we discuss the open challenges and research directions on designing a future intelligent road lighting system ${ }^{l}$.
\end{abstract}

Index Terms - Road lighting, system architecture, sensor networks, consumer electronics.

\section{INTRODUCTION}

Increasing awareness for climate change and impending shortage of energy sources accelerate the urge for more efficient light sources and lighting systems. Since lighting accounts for around $20 \%$ of the world's total electricity consumption, a massive changeover to more efficient lighting can help mitigating anthropogenic climate changes, increasing energy prices and maintenance costs. On the other hand, the main purpose of lighting is to serve people and their demands. Therefore, lighting systems must consider contextual information such as user profiles, the number of users and their behaviors via sensory data and interaction with users' $\mathrm{CE}$ devices (e.g., mobile phones). Currently, it is technically feasible to realize such an adaptive and interactive system thanks to the latest developments in lighting technology.

The fast advent of solid state light (SSL) sources provides more capabilities in terms of controlling light in comparison to classical light sources, such as fluorescent and incandescent lamps. It is possible to control the spectral, spatial, temporal polarization and color properties of light using an SSL source [1]. Furthermore, the high efficiency of solid-state lighting provides several benefits, in terms of energy consumption, overall carbon footprint, cost per lumen and lifetime [2]. Recent studies show that the use of light-emitting diodes

${ }^{1}$ Cagdas Atici, Tanir Ozcelebi and Johan J. Lukkien are with the Department of Mathematics and Computer Science, Eindhoven University of Technology, Den Dolech 2, 5612 AZ Eindhoven, The Netherlands (email: \{c.atici; t.ozcelebi; j.j.lukkien\}@tue.nl)

Contributed Paper

Manuscript received 03/23/11

Current version published 06/27/11

Electronic version published 06/27/11.
(LEDs) in a road lighting system is beneficial in terms of not only energy-efficiency but also visual effectiveness [3], [4]. In view of the goals that different countries and regions have laid out, this calls for, and enables, a much faster pace of efficiency improvement than is currently achieved, and requires paradigm shifts in both technology and application. Thus, the full potential of SSL is only to be reached on the system level and not on the component level.

Currently, outdated road lighting systems, which are very inefficient in terms of cost and energy consumption, are widely deployed all around the world. In these systems, luminaries are controlled with timers and/or photocells without any remote control or information retrieval options. There is no knowledge of exact amount of energy spent by each luminary and possible power leaks. Administrators rely on citizens to report any light faults since there is no central control or monitoring facility. Additionally, existing systems do not support any type of real-time information exchange or interactive communication for adapting to the changes in their surroundings. However, such a system requires not only the necessary communication infrastructure but also specific sensors to understand those changes.

Intelligent outdoor lighting solutions of the future must overcome these issues by utilizing a combination of SSL sources and suitable communication technologies. The main goals of such a system include reduction in the energy costs, greenhouse gas emissions, and installation and maintenance costs on the one hand, while retaining efficiency, reliability, user needs, public safety and scalability (in the number of poles) on the other. The system is said to be intelligent since light dimming commands are to be executed automatically based on the contextual information extracted from sensory data. The solution can be fully automated where an intelligent system reacts to changes using several sensors and actuators. Besides, the system can also be interactive where users interact with the system using CE devices, such as their mobile phones.

However, for such a system to work properly, the system architecture, the communication technologies and protocols chosen must support the requirements that arise from user scenarios and use cases. Furthermore, possible energy scavenging techniques and additional services must be investigated to maximize efficiency. Recently, various efforts have been made to design intelligent road lighting systems [5], [6]. In particular, realizing a road lighting testbed is of crucial importance to perform various subjective experiments, which has not been done in any of the aforementioned efforts. 
This paper is organized as follows. In Section II, we introduce the state-of-the-art road lighting solutions. In Section III, we describe the technical details of our road lighting testbed installation, which allows us to carry out user tests on a university campus street. Our aim is to investigate the relation between road lighting and safety feelings of pedestrians, where different brightness levels are compared [7]. In Section IV, we present a road map for an intelligent road lighting system while posing the open research directions and questions on this front. Finally, in Section V, we draw our conclusions.

\section{The State-OF-The ART Systems}

Recent press releases and reports demonstrate commercially available products on software-controlled road lighting front. All these systems follow a centralized architecture with a central terminal, a number of local branch nodes and a large number of actuators. Central terminal sends commands to actuators on each luminary post and also monitors the status of the system. Local branch nodes are responsible for sending commands received from the central terminal to actuators and for transmitting the feedback information from actuators to the central terminal. A general overview is as shown in Figure 1.

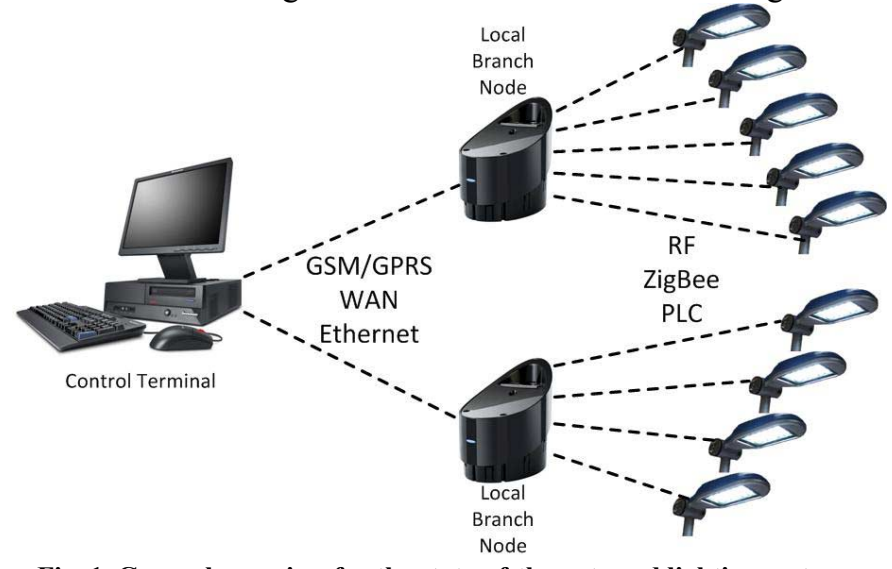

Fig. 1. General overview for the state-of-the-art road lighting systems.

Although the selected communication technology differs among these products, their common feature is that they include one long distance and one short distance transmission. One example [8] uses GPRS (General Packet Radio Service) technology for communication between the central server and the branch nodes, which are connected to luminaries by an RF (radio frequency) communication protocol. Another product uses WiMAC (Wireless Media and Control) protocol for communication between the actuators and the local nodes while the system utilizes a web-based central management system [8]. The system in [9] includes ZigBee modules for linking the luminary controllers to a local hub where these hubs communicate with the central server using cellular and internet connection. In [10], a cost-effective solution for monitoring, controlling and managing road lighting is presented, where power-line communications is used for information exchange between the luminaire controllers and the central controller. The networking technologies used by these systems are summarized in Table 1.
TABLE I

COMPARISON FOR THE STATE-OF-THE-ART ROAD LIGHTING SYSTEMS

Communication between actuators \& local/central node

Proprietary RF

Proprietary RF

ZigBee

Power-line communications

Communication between central node \& server GPRS Cellular \& Internet

Wide Area Network/Internet Ethernet/Internet

One important observation is that, almost all of the commercial products aim to decrease the energy consumption of the overall system by adjusting the brightness levels while ignoring user satisfaction and user interaction.

\section{OUR TESTBED}

We establish a software-controlled road lighting testbed in order to perform experiments that can help us to identify user requirements and the possible future challenges on this front. Apart from being an optimal or efficient solution to the intelligent road lighting problem, we build a cost-effective and functional testbed in order to conduct necessary experiments. We focus on the relationship between the users' feeling of safety at night and the brightness patterns of the luminaries. Here, we present the technical details of our testbed.

\section{A. Problem Description}

Consider pedestrians walking on a road, where there are a number of evenly spaced luminary poles. Assuming an average pedestrian walking speed of $1.25 \mathrm{~m} / \mathrm{s}$ and a separation of $25 \mathrm{~m}$ between every two adjacent luminary poles, it takes 20 seconds for a pedestrian to walk from one pole to the next.

An intelligent road lighting system must adjust the brightness level of each luminary in this interval of 20 seconds based on the position and direction of the pedestrian subject. This means that light brightness adjustment commands need to propagate to all poles involved in less than 20 seconds. As a worst case scenario, assume the system needs to adjust 10 poles simultaneously and turn each luminary from a fully off state to a $100 \%$ on state. However, a luminary turning from fully off to fully on state in a single step does not look natural to a pedestrian user. In order to improve user satisfaction, switching between any two states must be smooth, i.e. using a number of consecutive intermediate states. Assuming a fine quantization with step sizes of $10 \%$ brightness, it requires 10 messages per pole to do this switching. Therefore, the system needs to support successful transmissions of 100 messages in 20 seconds in the worst case scenario, such that an area of 125 meters in both forward and backward directions can be dynamically lit within the specified time interval.

\section{B. System Architecture}

Following the above analysis, we can state that the use of power-line communications (PLC) for sending commands to the luminary poles is feasible. The bandwidth of power-line is theoretically limited to a maximum of 8 messages/sec [11]. Due to the transmission errors and packet losses, power-line operates at an average throughput of 5 messages/sec in practice, which is sufficient even for the worst case scenario 
described previously. Using PLC also reduces installation costs significantly, since no additional wiring is required for establishing the infrastructure.

We build the testbed with a control PC, a segment controller (SC), 10 outdoor luminaire controllers (OLCs), i.e., 1 per luminary, and 10 luminaries. The $\mathrm{SC}$ and the OLC communicate through power-line wires while the control PC connects to the SC via Ethernet. The PC graphical user interface (GUI) is used for monitoring and sending commands to the SC and for collecting and analyzing the feedback data received from the SC. The system architecture is depicted in Figure 2. The technical details of a similar system model using PLC is also described in [11] and references therein.

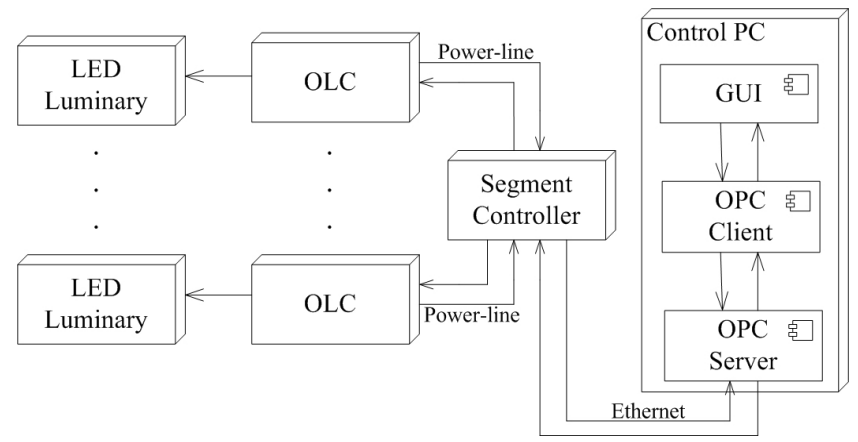

Fig. 2. System architecture diagram (deployment view)

Each luminary used in the testbed includes 84 LEDs with a total power consumption of $106 \mathrm{~W}$ and light output of 8820 $\mathrm{lm}$ at $100 \%$ brightness. Using a simple setup, we measure the voltage characteristics of the LED luminary in a dark room where all the effects of the interfering lights are excluded. Figure 3 presents the relationship between the luminance value of the luminary and the voltage supplied by the OLC. As expected, illuminance (in lux) increases linearly with the supplied voltage after a certain voltage threshold.

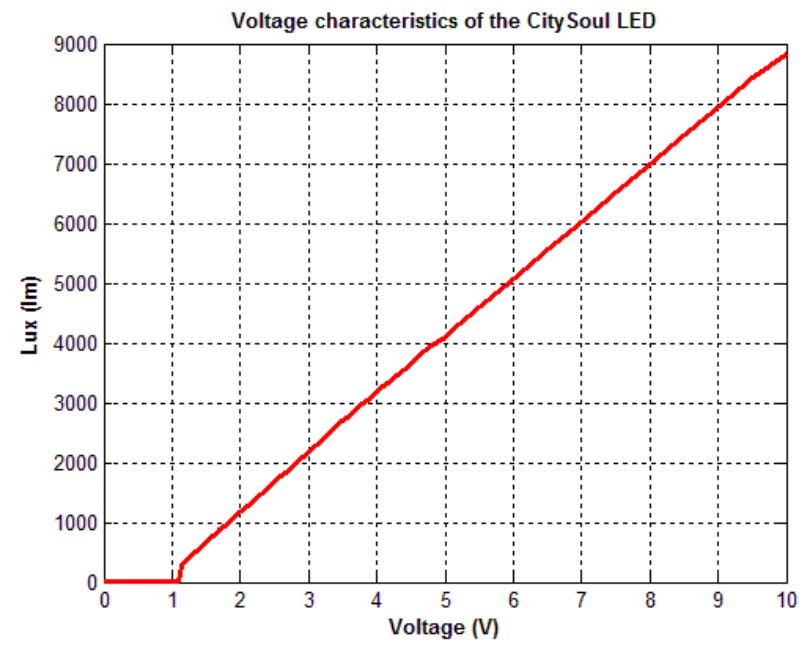

Fig. 3. Lux versus voltage graph for the CitySoul LED luminary.

The SC is responsible for monitoring and controlling the OLCs. Among the various functionalities of the SC, such as scheduling, alarming and logging, we use only the two basic parameters required to control the luminary brightness levels.
These parameters are 'state' and 'value'. The former is a boolean variable that defines the on-off state of the luminary while the latter is an integer value in the range 0-100, defining the brightness percentage of the luminary. The brightness of each luminary is adjusted by the OLCs, where a voltage value between $1-10 \mathrm{~V}$ is set according to the requested brightness value as shown in Figure 3.

In our testbed, we use an OPC - Object Linking and Embedding (OLE) for Process Control - client/server application pair for the communication between the SC and the PC in the control room. OPC is a widely accepted plugand-play software application, which provides an open standard for interoperability [12]. One can access the SC using a web-based GUI via HTTP, or using software with a GUI as in Figure 4, which shows a snapshot of our GUI. This program includes an OPC client and allows a human operator to switch between different scenarios effectively. The brightness levels can be read from a file or the operator can select a brightness pattern from predetermined scenarios. It is possible to set the starting time, running time and step size for dimming up and down.

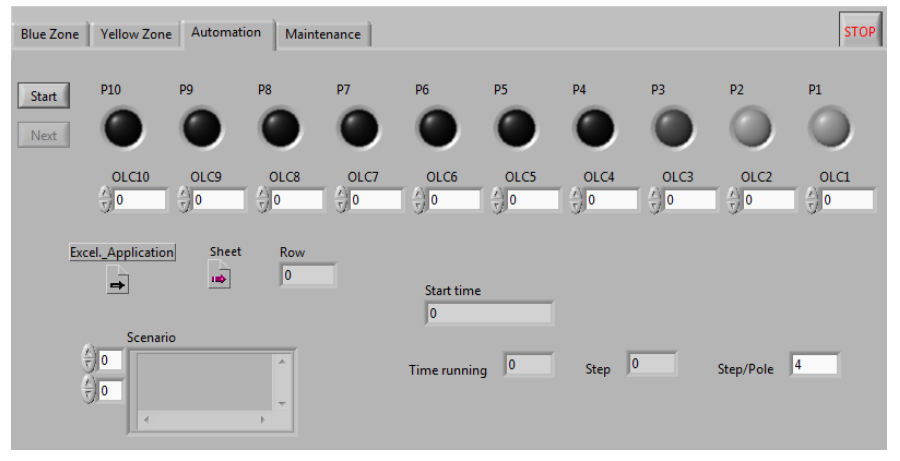

Fig. 4. GUI used in the tested for road lighting experiments.

\section{RESEARCH CHALlenges AND DIRECTIONS}

In this section, we summarize the challenges and the possibilities for future road lighting systems regarding user experience, system design, energy scavenging and additional services.

\section{A. User Experience}

An intelligent road lighting system must go beyond simply illuminating people and their vicinity. Feeling of safety is a very important performance indicator. Studies [13], [14] show that improved road lighting reduces crime and fear during the night hours. Therefore, the relationship between the lighting patterns and the feeling of comfort and safety at night from a user perspective should be investigated. For this purpose, we conducted a series of experiments using our testbed.

The experiments are conducted on De Zaale street on the Eindhoven University of Technology campus where 10 luminaries are approximately evenly spaced $(25 \mathrm{~m}$ distance between adjacent poles). The LED luminaries have been installed using a metal fork along with the traditional HID luminaries. A close-up photo of the LED luminaries and a view from De Zaale are shown in Figure 5. 


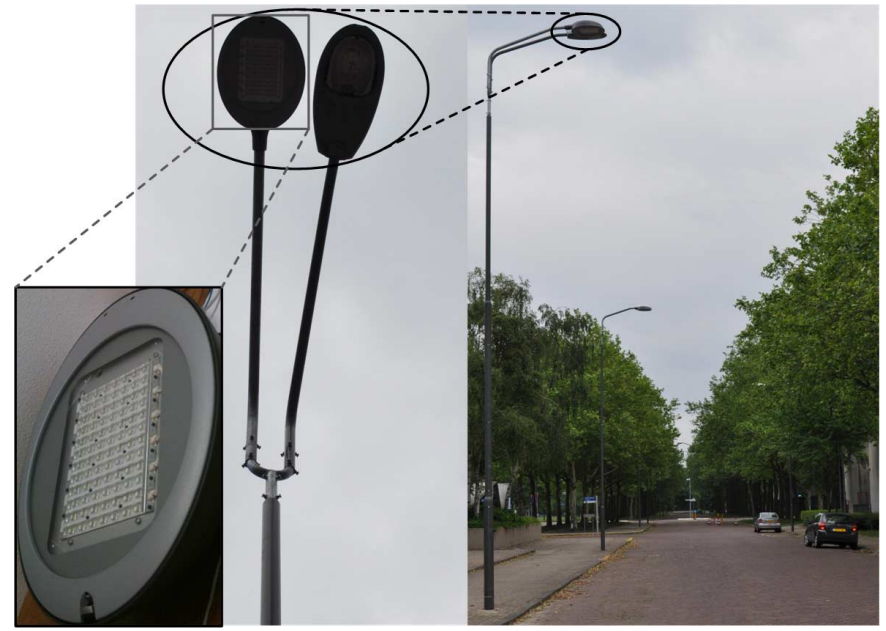

Fig. 5. LED and HID luminaries (left), a view from the testbed (right).

The schematic of the experiment is given in Figure 6. The small circles and squares denote the positions of the LED luminaries used in our experiments and the interfering traditional luminary poles, respectively. The red star in the center shows the initial position of the subjects who rated different scenarios according to the perceived safety [7]. After some initial experiments, we observed that the interfering luminary poles in the surrounding degrade the reliability of the questionnaire. In order to avoid external light interference, we installed additional OLCs to each interfering luminary pole, and connected them to a second SC. Before each experiment, interfering light sources are turned off via the PC GUI.

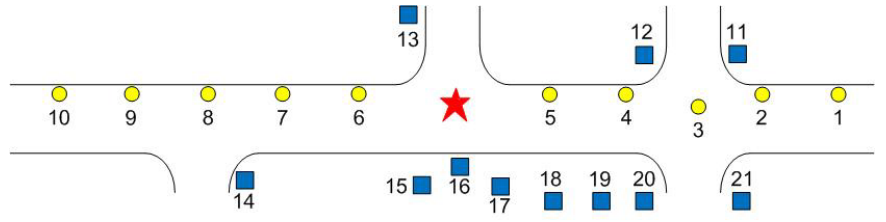

Fig. 6. Schematic of the experiment on the testbed.

In the experiments, all the possible combinations of three different scenarios for both left and right sides of the street are compared: flat (all luminaries at $40 \%$ dimming), increasing/decreasing (with $20 \%$ step sizes) [7]. In the first series of experiments, i.e. the static pedestrian scenario, subjects rated the questionnaire by standing on the initial location shown by the star in Figure 6. In the second series, dynamic pedestrian scenario, same brightness level combinations are implemented according to the position of the walking pedestrian by illuminating a total of five poles, the nearest pole and two poles in both forward and backward directions. The results of the static scenario show that an increasing brightness pattern makes people feel safer while a triangular lighting pattern (the nearest pole has the maximum illumination and brightness decays away from the user) is the best rated one in the dynamic scenario.

Although we present results of our study for pedestrian case, other forms of mobility cases, such as cyclist and vehicular, should also be analyzed. The requirements for a cyclist or a car driver are different than the pedestrian user. For example, a car driver needs to have the maximum visibility of the road and the objects closer to the road instead of the safety feeling. In addition, weather conditions affect the vision of a car driver differently than a cyclist or a pedestrian. Thus, an intelligent road lighting system should be aware of the different classes of users and take into account the disparities among their needs. A set of recommendations are given in [15] for improved performance with respect to user satisfaction.

Besides switching between scenarios, road lighting can be interactive, e.g. users interacting with the system using their mobile phones. According to [16], decision mechanisms can be further improved in an interactive system. When the system is equipped with further sensing and actuation technology, it can respond to particular situations such as accidents, dangerous situations and changes in weather. Complex responses require a fully- or semi-distributed self-contained system since centralized control will no longer be feasible.

Traditional road lighting systems are thought to be the main cause of light pollution [17], which affects visibility of stars with naked eye (bad for causal viewers and astronomers) and disorients the life of various animals living in the vicinity [18]. Future road lighting systems can help diminish light pollution. A thorough analysis and methods to deal with light pollution caused by luminaries are presented [19].

\section{B. System Architecture and Networking Technology}

Assuming an actuator is installed on each lamp post, all the lamps in the system can be controlled independently. Then, the road lighting problem in hand includes both long and short distance communications. In all of the commercially available solutions and also the proposed solutions in the literature, there is one central terminal and several controllers. In general, the distance between the central terminal and the controllers is very large when compared to the distance between each controller and the actuators associated with controllers. For the long distance communication problem, it is common to use Ethernet, WiMaX, cellular communication (GPRS, $3 \mathrm{G}$ or others), while PLC, WiFi, ZigBee and other types of RF communication are typically used for short distances.

The choice of the system architecture, a centralized or semiautonomously distributed fashion, is of crucial importance. A centralized architecture using power-line communications is very cost-efficient since it uses the already installed power cables as the communication medium. A SC, which receives commands from a host controller (e.g. a PC), acts as the gateway node and sends commands to the actuators in the system. However, PLC is very limited in terms of bandwidth and is prone to high signal attenuation and distortion [20]. The communication between the host and the SC can be Ethernet, $\mathrm{WiFi}$, GPRS or $3 \mathrm{G}$ as long as the controller supports these standards. A communication method by GPRS domain name is suggested in [21]. Furthermore, a mechanism with CRC (Cyclic Redundancy Check) coding is introduced to increase the efficiency and the security of the system. Another approach is the use of master and slave electronic ballasts for receiving commands from the central terminal [22]. However, the maximum number of slaves that can be connected to one master 
node is not mentioned in this study. Another study [23] presents a prototype control terminal which consists of one CDMA and one ZigBee transceiver for long and short distance communication, respectively. In [24], use of ZigBee protocol is proposed where a total of 65,535 lamps can be connected to one controller. The communication between the host terminal and the controllers is done via GPRS. The information from GRPS and ZigBee networks can be exchanged with each other using an interface module. The module includes a microprocessor, in addition to GRPS and ZigBee transceivers.

For a semi-autonomous distributed network approach, we can treat the road lighting system as a wireless sensor actuator network and implement a loader which commissions several services to all the actuators via gateway nodes [25]. Although [26] presents a lightweight solution for smart spaces composed of low capacity nodes using ZigBee, a similar solution can be implemented for a road lighting system in order to provide interactivity and deal with the connectivity problems. The proposed open service architecture for sensors (OSAS) enables automatic discovery of components and monitoring services and resources at run-time. These properties dramatically ease the installation phase for a road lighting system since only one module, including at least an actuator and a transceiver, should be placed on each and every lamp post. In [27], ATmega128 chips are used on each pole and multi-hopping is supported. Here, an efficient routing protocol is required to adapt the changes in connectivity, such as broken links, in order to preserve the integrity of the network. To illustrate, [28] points out the problems in the routing mechanism of ZigBee in case of failures and proposes a new routing algorithm utilizing the GPS information since lamp posts have fixed position.

The types of sensors and sensory data, as well as the required post-processing for information extraction must be further explored for road lighting. Note that, without any processing of sensory data, communication can easily become a dominating factor in energy consumption. Thus, on-the-node processing, where a part of the collected sensory data is processed locally before transmission can minimize the overall energy consumption and reduce the network load. Besides, the communication protocols have to be energyefficient while satisfying the required latency, robustness and bandwidth constraints. To illustrate, researchers work on analyzing the energy consumption in different modes of the ZigBee protocol and ways to minimize this usage [29], [30].

Last but not the least, the lighting system must be secure enough to prevent any type of intrusion. Although the security and reliability concerns in the use of wireless technologies are presented in [31], there is no work on building secure road lighting systems. However, [32] can be a good starting point in order to implement necessary security measures in a future road lighting systems.

\section{Energy Scavenging}

An intelligent road lighting system may utilize solar energy [33], wind energy [34], [35] or other forms of energy scavenging in order to further reduce the energy demands. A very recent study presents an optimized hybrid system design for road lighting [36]. In this work, authors described the cost optimization for a coupling of photovoltaic generator with fuel cell and battery.

\section{Additional Services}

The future road lighting systems should also provide additional services besides illuminating the streets and pathways. If equipped with necessary sensors, street poles can give information regarding weather conditions (humidity and temperature), road status (traffic intensity and maintenance operations) and failures. Additionally, they can provide information about the road surface condition [37], any type of degradations (on the road or the barriers) and physical objects on the roads, such as rocks, small car parts and wild animals that may cause an accident. Such additional services will not only reduce the time and effort spent for maintenance but also degrade the possibility of accidents and improve the reaction time in case of emergency situations.

\section{Conclusion}

Designing an intelligent road lighting system is a necessity because of the inefficiencies in the traditional systems. These old systems not only consume huge amounts of energy but also are incapable of reacting to the changes in their environment.

We built our own intelligent road lighting system to carry out user experiments on a real dynamic testbed and identify social and technical challenges. Therefore, it was not our main goal to optimize such a system and our specific design choices were mainly driven by ease of installation and cost efficiency. The speed of our testbed is limited by the PLC speed. The PLC infrastructure allowed us to do experiments at pedestrian speeds. However, as future work, we plan to use wireless inter-hop communication to facilitate user tests at vehicular speeds.

While building a software-controlled road lighting testbed, we have identified various research problems that must be addressed in detail and presented them in this paper. None of the commercial and noncommercial solutions that we have come across so far presents a dynamic intelligent road lighting system that takes user experience, and specifically the feeling of safety at night, into account. We investigated intelligent road lighting systems from user experience, technology, energy perspectives and proposed useful services that can be enjoyed using the same infrastructure.

\section{REFERENCES}

[1] J. Kim and E. Schubert, "Transcending the replacement paradigm of solid-state lighting," Opt. Exp., vol. 16, no. 26, pp. 21835-21842, 2008.

[2] E. Schubert and J. Kim, "Solid-State Light Sources Getting Smart," Science, vol. 308, no. 5726, pp. 1274-1278, 2005.

[3] L. Halonen and M. Puolakka, "The Use of LEDs and Applications of the New Mesopic Design in Road Lighting," Solid-State and Organic Lighting, 2010.

[4] F. Li, D. Chen, X. Song, and Y. Chen, "LEDs: A Promising EnergySaving Light Source for Road Lighting," Proc. Power and Energy Eng. Conf. (APPEEC), pp. 1-3, 2009.

[5] L. Guo, M. Eloholma, and L. Halonen, "Intelligent road lighting control systems," Report 50, Helsinki University of Technology, 2008. 
[6] P. R. Boyce, S. Fotios, and M. Richards, "Road lighting and energy saving," Lighting Res. \& Techn., vol. 41, no. 3, pp. 245-260, 2009.

[7] Y. A. W. de Kort, A. Haans, L. Geerdinck, D. van Gennip, M. Horst, and J. Servaes, "Psychological building blocks for dynamic road lighting: Understanding light's role in feelings of safety at night," Proc. of LS12WLED3, TU/e, 2010.

[8] F. Pearce, "RMS and CMS for Road Lighting - a Technology Overview", Lighting Journal, vol. 74, no. 2, pp. 38-42, 2009.

[9] D. Taub, E. Lupton, R. Hinman, S. Leeb, J. Zeisel, and S. Blackler, "The Escort System: A Safety Monitor for People Living with Alzheimer's," IEEE Pervasive Computing, 2010.

[10] P. Edwards, R. Grosvenor, and P. Prickett, "A Review of Lamp Condition Monitoring Technologies," Lighting Journal, v. 71, no. 5, pp. 31-37, 2006.

[11] S.K. Cho and V. Dhingra, "Street Lighting Control based on LonWorks Power Line Communication," Proc. IEEE Int. Symp. on Power Line Comm. and Its App. (ISPLC), pp. 396-398, April 2008.

[12] X. Hong and W. Jianhua, "Using standard components in automation industry: A study on OPC Specification," Computer Standards \& Interfaces, vol. 28, no. 4, pp. 386-395, 2006.

[13] K. Painter, "The influence of street lighting improvements on crime, fear and pedestrian street use, after dark," Landscape and Urban Planning, vol. 35, no. 2-3, pp. 193-201, 1996.

[14] D.P. Farrington and B.C. Welsh, "Effects of improved street lighting on crime: a systematic review," Home Office Research Study 251, Development and Statistics Directorate, Aug. 2002.

[15] M. Kostic and L. Djokic, "Recommendations for energy efficient and visually acceptable street lighting," Energy, vol. 34, no. 10, pp. 15651572,2009

[16] J.S. Sandhu, A.M. Agogino, and A.K. Agogino, "Wireless Sensor Networks for Commercial Lighting Control: Decision Making with Multi-agent Systems," AAAI workshop on sensor networks, pp. 131-140, 2004.

[17] C. Shaflik, "Environmental Effects of Roadway Lighting," International Dark-Sky Association (IDA) Information Sheet, vol. 125, pp. 1-12, 1997.

[18] T. Longcore and C. Rich, "Ecological ligh pollution," Frontiers in Ecology and the Environment, vol. 2, no. 4, pp. 191-198, 2004.

[19] P. Cinzano, "Light pollution by luminaries in roadway lighting," CIE Division TC4-21, pp. 1-8, 2003.

[20] V.C. Gungor and F.C. Lambert, "A survey on communication networks for electric system automation," Computer Networks, vol. 50, no. 7, pp. 877-897, 2006.

[21] D. Liu, S. Qi, T. Liu, S. Yu, and F. Sun, "The Design and Realization of Communication Technology for Street Lamps Control System", Proc. Int. Conf. on Comp. Science \& Education (ICCSE), pp. 259-262, Nov. 2009.

[22] G.B. Maizonave, F.S. Dos Reis, J.C.M. Lima, A.J. Bombardieri, F.E. Chiapetta, G.B. Ceccon, R.R.N. Souza, Jr. Tonkoski, and R.W. Dos Reis, "Integrated System for Intelligent Street Lighting", Proc. IEEE Int, Symp. on Industrial Elec. (ISIE), pp. 721-726, July 2006.

[23] J.D. Lee, K.Y. Nam, S.H. Jeong, S.B. Choi, H.S. Ryoo, and D.K. Kim, "Development of ZigBee based Street Light Control System," Proc. IEEE Power Systems Conf. and Expo. (PSCE), pp. 2236-2240, Oct. 2006

[24] L. Li, X. Chu, Y. Wu, and Q. Wu, "The Development of Road Lighting Intelligent Control System Based on Wireless Network Control," Proc. IEEE Int. Conf. on Elec. Comp. Tech. (ICECT), pp. 353-357, Feb. 2009.

[25] M. Mendalka, M. Gadaj, L. Kulas, and K. Nyka, "WSN for intelligent street lighting system," Proc. Int. Conf. on Information Tech. (ICIT), pp. 99-100, June 2010

[26] T. Ozcelebi, J. Lukkien, R. Bosman, and O. Uzun, "Discovery, Monitoring and Management in Smart Spaces Composed of Low Capacity Nodes," IEEE Tran. on Cons. Elec., vol. 56, no. 2, pp. 570578, May 2010.

[27] C. Jing, D. Shu, and D. Gu, "Design of Streetlight Monitoring and Control System Based on Wireless Sensor Networks," Proc. IEEE Industrial Elec. and App. (ICIEA), pp. 57-62, May 2007.

[28] G.W. Denardin, C.H. Barriquello, R.A. Pinto, M.F. Silva, A. Campos, and R.N. do Prado, "An Intelligent System for Street Lighting Control and Measurement," Proc. IEEE Industry App. Society Annual Meeting (IAS), pp. 1-5, Oct. 2009.
[29] B. Bougard, F. Catthoor, D.C. Daly, A. Chandrakasan, and W. Dehaene, "Energy Efficiency of the IEEE 802.15.4 Standard in Dense Wireless Microsensor Networks: Modeling and Improvement Perspectives," Proc. Design, Automation, and Test in Europe, pp. 196-201, 2005.

[30] C. Mathuna, T. O’Donnell, R.V. Martinez-Catala, J. Rohan, and B. O'Flynn, "Energy scavenging for long-term deployable wireless sensor networks," Talanta, vol. 75, no. 3, pp. 613-623, 2008.

[31] F. Cleveland, "Use of Wireless Data Communications in Power System Operations," Proc. IEEE Power Systems Conf. \& Expo. (PSCE), pp. 631-640, Oct. 2006

[32] P. Baronti, P. Pillai, V.W.C. Chook, S. Chessa, A. Gotta, and Y.F. Hu, "Wireless sensor networks: A survey on the state of the art and the 802.15.4 and ZigBee standards," Computer Communications, vol. 30, no. 7, pp. 1655-1695, 2007.

[33] M.A. Dalla Costa, L. Schuch, L. Michels, C. Rech, J.R. Pinheiro, and G.H. Costa, "Autonomous Street Lighting System based on Solar Energy and LEDs," Proc. IEEE Int. Conf. on Industrial Tech. (ICIT), pp. 1143-1148, March 2010.

[34] A. Holt and I.J. Pengelly, "ITS and Renewable Energy," Proc. Road Transport Information and Control (RTIC) and ITS United Kingdom Members' Conf. (IET), pp. 1-6, May 2008.

[35] L. Liu and Z. Wang, "The development and application practice of wind-solar energy hybrid generation systems in China," Renewable and Sustainable Energy Reviews, vol. 13, no. 6-7, pp. 1504-1512, 2009.

[36] J. Lagorse, D. Paire, and A. Miraoui, "Sizing optimization of a standalone street lighting system powered by a hybrid system using fuel cell, PV and battery," Renewable Energy, vol. 34, no. 3, pp. 683-691, 2009.

[37] T.F. Golob and W.W. Recker, "Relationships Among Urban Freeway Accidents, Traffic Flow, Weather, and Lighting Conditions," Journal of Transportation Engineering, vol. 129, no.4, pp. 342-353, 2003.

\section{BIOGRAPHIES}

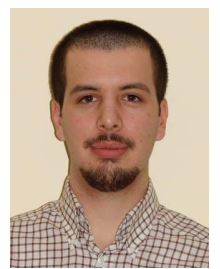

Çağdaş Atıcı received his B.Sc. and Ph.D. degrees from Koç University, Istanbul, Turkey both with high honors. He received the Dean's Award in 2005 due to his high scholarly achievements. He was also a recipient of the Tübitak Ph.D. Fellowship. In 2007, he won the best Academic Project prize by Turkcell in "Mobil Gelecek" competition. He is listed in Marquis Who's Who in the World 2010. He is now a postdoctoral researcher in System Architecture \& Networking Group at Eindhoven University of Technology. His research interests include wireless system design, mobile adhoc networks, video broadcasting and wireless sensor networks.

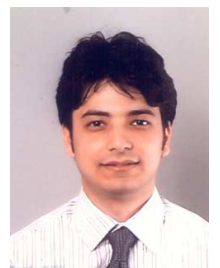

Tanır Özçelebi received the B.Sc. degree in electrical and electronics engineering from Bilkent University, Ankara, in 2002 and the Ph.D. degree in electrical engineering from Koç University, Istanbul, in 2006. In 2006, he joined the System Architecture and Networking Research group at Eindhoven University of Technology as a postdoctoral researcher, and became an assistant professor in the same research group in 2008. His research interests are resource and service discovery and management, quality of service management for networked systems and design of smart distributed systems.

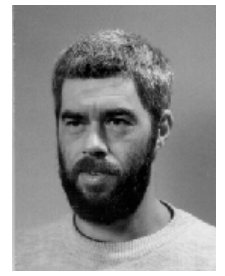

Johan J. Lukkien is head of the System Architecture and Networking Research group at Eindhoven University of Technology since 2002. He received M.Sc. and Ph.D. from Groningen University in the Netherlands. In 1991 he joined Eindhoven University after a two years leave at the California Institute of Technology. His research interests include the design and performance analysis of parallel and distributed systems. Until 2000 he was involved in large-scale simulations in physics and chemistry. Since 2000, his research focus has shifted to the application domain of networked resource-constrained embedded systems. Contributions of the SAN group are in the area of component-based middleware for resource-constrained devices, distributed coordination, Quality of Service in networked systems and schedulability analysis in real-time systems. 